\title{
Transforming Positions and Velocities between the International Terrestrial Reference Frame of 2000 and North American Datum of 1983
}

\author{
Tomás Soler, M.ASCE, ${ }^{1}$ and Richard A. Snay ${ }^{2}$
}

\begin{abstract}
In December 2001, Natural Resources Canada and the U.S. National Geodetic Survey (NGS) jointly adopted values for a set of 14 parameters for transforming positional coordinates and velocities between the International Terrestrial Reference Frame of 2000 (ITRF00) and the North American Datum of 1983 (NAD 83). Seven of these parameters characterize the variation with respect to time of the standard seven parameters (three shifts, three rotations, and a differential scale). In March 2002, the NGS updated the NAD 83 positional coordinates for all continuously operating reference stations to be consistent with their corresponding ITRF00 coordinates at an epoch date of 2002.00. Also, the NGS has incorporated these adopted values for the 14 transformation parameters into software packages, such as HTDP (horizontal time dependent positioning) for transforming positional coordinates across time and between reference frames, and OPUS (On-line Positioning User Service) for processing global positioning system data. Both HTDP and OPUS are available through the NGS Tool Kit (http://www.ngs.noaa.gov/TOOLS/).
\end{abstract}

DOI: 10.1061/(ASCE)0733-9453(2004)130:2(49)

CE Database subject headings: Datum; North America; Global positioning; Geodetic surveys; Velocity.

\section{Introduction}

A significant breakthrough in the geosciences field was accomplished with the introduction, more than two decades ago, of the Global Positioning System (GPS). The GPS deserves credit for substantially increasing our knowledge of geocentric terrestrial coordinate systems. It represents a substantial leap forward from the regionally defined coordinate systems of pre-GPS vintage, for example, the North American Datum of 1927 (NAD 27) and the South American Datum of 1956 (SAD 56). The accuracy currently available in geocentricity, orientation, and scale of terrestrial coordinate frames will be difficult to improve significantly in the foreseeable future.

This achievement has been made possible primarily by refinements in the precise ephemerides (orbits) disseminated by the International GPS Service (IGS). These IGS orbits have been derived from the GPS data recorded at numerous ground-based stations, and they currently provide positions for the GPS satellites at the $5 \mathrm{~cm}$ level (Springer and Hugentobler 2001). Moreover, the IGS supplies, via the Internet, predicted orbits, referred to as "ultra-rapid" orbits, that give 3D estimates of satellite positions with a standard error in the $30-$ to $40-\mathrm{cm}$ range. This precision

${ }^{1}$ Chief, Global Positioning System Branch, National Geodetic Survey, NOS, National Oceanic and Atmospheric Administration, 1315 East-West Hwy., Silver Spring, MD 20910. E-mail: Tom.Soler@noaa.gov

${ }^{2}$ CORS Program Manager, National Geodetic Survey, NOS, National Oceanic and Atmospheric Administration, 1315 East-West Hwy., Silver Spring, MD 20910. E-mail: Richard.Snay@noaa.gov

Note. Discussion open until October 1, 2004. Separate discussions must be submitted for individual papers. To extend the closing date by one month, a written request must be filed with the ASCE Managing Editor. The manuscript for this paper was submitted for review and possible publication on August 29, 2002; approved on April 18, 2003. This paper is part of the Journal of Surveying Engineering, Vol. 130, No. 2, May 1, 2004. (CASCE, ISSN 0733-9453/2004/2-49-55/\$18.00. represents a great improvement over the U.S. Department of Defense (DoD) broadcast orbits, the standard error for whose satellite positions is about $2.7 \mathrm{~m}$ (Springer and Hugentobler 2001).

A large amount of effort has been expended on exploiting the advent of precise positioning. Several countries have readily deployed permanent GPS satellite tracking networks to establish a fundamental framework for differential GPS positioning at subcentimeter accuracies. GPS campaigns have proliferated in recent years, and new, superior continental reference systems have evolved around the globe. These include the European Terrestrial Reference Frame of 1989 (ETRF89), the Geocentric Datum of Australia 1994 (GDA94), the North American Datum of 1983 (NAD 83), and the South American Geocentric Reference System (SIRGAS). More information about the frames used in these particular datums is given in Soler and Marshall (2003). Subsequently, local networks have been densified and rigorous control for mapping and surveying has been extended. Also, as a result of more sophisticated national geoid height models-so critical for GPS leveling - the GPS now affords a more economical alternative to differential leveling for many heighting applications.

GPS users can now determine the 3D positional coordinates of a point with centimeter accuracy relative to a control station located several hundred kilometers away (Eckl et al. 2001). As an added incentive, a profusion of continuously operating reference stations (CORS) exists today that are operated for various different applications. Such is the case for the National CORS network, which is managed by the National Geodetic Survey (NGS) in cooperation with more than 100 organizations, each of which operates at least one CORS. The National CORS network as of March 2004 contained 459 permanently monumented GPS base stations, and it is growing at a rate of about 7 stations per month. Positional coordinates of the National CORS sites form the foundation for the National Spatial Reference System (NSRS), the backbone for all geodesy, surveying, and mapping applications in the United States. Not only is the National CORS network be- 
coming a natural tool for accurate 3D positioning in the country, but its GPS data holdings are used by a plethora of investigators interested in applications as diverse as ionospheric research, crustal motion, water vapor studies, and aerial mapping (Snay et al. 2002). For more information about the CORS program, consult the Web address http://www.ngs.noaa.gov/CORS/.

Positional coordinates for the stations defining the NSRS are expressed relative to the North American Datum of 1983, NAD 83 for short. These NAD 83 coordinates are in turn defined through a Helmert (similarity) transformation from coordinates expressed relative to the International Terrestrial Reference Frame of 2000 (ITRF00), the most accurate geocentric reference frame currently available.

It should be pointed out here that recent studies by Merrigan et al. (2002) indicate that ITRF00 and World Geodetic System of 1984 (WGS 84) (G1150) are essentially identical. ITRFxx frames, where $\mathrm{xx}$ denotes the last two digits identifying the solution, are created under international sponsorship and satisfy stringent accuracy requirements for various modern space-based geodetic techniques. Associated with each ITRFxx frame is a specified epoch date to which the published positions correspond. The position for some other date might differ due to phenomena such as crustal motion. The epoch associated with ITRF00 is $t_{0}$ $=1997.00$ (January 1, 1997). Every well-defined reference frame must specify a velocity for each point to transform its positional coordinates from epoch $t_{0}$ to any other arbitrary epoch $t$.

The U.S. National Geodetic Survey (NGS) determines positional coordinates for CORS sites by exploiting the most up-todate and advanced GPS methodologies and software. Initially, these coordinates are rigorously computed in ITRF00. Introductory information about ITRF frames can be found in Boucher and Altamimi (1996) and Snay and Soler (2000b). The final step in CORS processing is to transform the ITRF00 coordinates into NAD 83 coordinates. This requires implementing a set of transformation equations, as will be explained in the following section.

\section{Transforming Positional Coordinates}

Let $x(t)_{\mathrm{NAD} 83}, y(t)_{\mathrm{NAD} 83}$, and $z(t)_{\mathrm{NAD} 83}$ denote the NAD 83 positional coordinates for a point at time $t$ as expressed in a 3D Cartesian Earth-centered, Earth-fixed coordinate system. These coordinates are expressed as a function of time to reflect the reality of the crustal motion associated with plate tectonics, land subsidence, volcanic activity, postglacial rebound, and so on. Similarly, let $x(t)_{\mathrm{ITRF}}, y(t)_{\mathrm{ITRF}}$, and $z(t)_{\mathrm{ITRF}}$ denote the ITRFXx positional coordinates for this same point at time $t$. The given ITRFxx coordinates are related to their corresponding NAD 83 coordinates by a Helmert transformation that is approximated by the following equations:

$$
\begin{aligned}
x(t)_{\mathrm{NAD} 83}= & T_{x}(t)+[1+s(t)] \cdot x(t)_{\mathrm{ITRF}}+\omega_{z}(t) \cdot y(t)_{\mathrm{ITRF}} \\
& -\omega_{y}(t) \cdot z(t)_{\mathrm{ITRF}} \\
y(t)_{\mathrm{NAD} 83}= & T_{y}(t)-\omega_{z}(t) \cdot x(t)_{\mathrm{ITRF}}+[1+s(t)] \cdot y(t)_{\mathrm{ITRF}} \\
& +\omega_{x}(t) \cdot z(t)_{\mathrm{ITRF}} \\
z(t)_{\mathrm{NAD} 83}= & T_{z}(t)+\omega_{y}(t) \cdot x(t)_{\mathrm{ITRF}}-\omega_{x}(t) \cdot y(t)_{\mathrm{ITRF}} \\
& +[1+s(t)] \cdot z(t)_{\mathrm{ITRF}}
\end{aligned}
$$

Here $T_{x}(t), T_{y}(t)$, and $T_{z}(t)$ are translations along the $x$-, $y$-, and $z$-axes, respectively; $\omega_{x}(t), \omega_{y}(t)$, and $\omega_{z}(t)$ are counterclockwise rotations about these same three axes; and $s(t)$ is a differ- ential scale change between ITRFxx and NAD 83. These approximate equations suffice because the three rotations usually have rather small magnitudes. Note that each of these seven quantities is represented as a function of time because improvements in space-based geodetic techniques have enabled us to detect their time-related variations with some degree of accuracy. A decade ago such time-dependent variations were poorly known and often overlooked [for example, Soler and Hothem $(1988,1989)]$. These time-dependent variations are assumed to be mostly linear, so that the quantities may be expressed by the following equations:

$$
\begin{gathered}
T_{x}(t)=T_{x}\left(t_{0}\right)+\dot{T}_{x} \cdot\left(t-t_{0}\right) \\
T_{y}(t)=T_{y}\left(t_{0}\right)+\dot{T}_{y} \cdot\left(t-t_{0}\right) \\
T_{z}(t)=T_{z}\left(t_{0}\right)+\dot{T}_{z} \cdot\left(t-t_{0}\right) \\
\omega_{x}(t)=\left[\varepsilon_{x}\left(t_{0}\right)+\dot{\varepsilon}_{x} \cdot\left(t-t_{0}\right)\right] \cdot m_{r} \\
\omega_{y}(t)=\left[\varepsilon_{y}\left(t_{0}\right)+\dot{\varepsilon}_{y} \cdot\left(t-t_{0}\right)\right] \cdot m_{r} \\
\omega_{z}(t)=\left[\varepsilon_{z}\left(t_{0}\right)+\dot{\varepsilon}_{z} \cdot\left(t-t_{0}\right)\right] \cdot m_{r} \\
s(t)=s\left(t_{0}\right)+\dot{s} \cdot\left(t-t_{0}\right)
\end{gathered}
$$

where $m_{r}=4.84813681 \times 10^{-9} \equiv$ conversion factor from milliarc seconds (mas) to radians.

Here, $t_{0}$ denotes a fixed, prespecified time of reference, commonly called the "reference epoch date." Hence the seven quantities $T_{x}\left(t_{0}\right), T_{y}\left(t_{0}\right), \ldots, s\left(t_{0}\right)$ are all constants. The seven other quantities, $\dot{T}_{x}, \dot{T}_{y}, \ldots, \dot{s}$, which represent rates of change with respect to time, are also assumed to be constants.

In 1997, Natural Resources Canada (NRCan) and the NGS jointly adopted values for these 14 constants to transform ITRF96 positional coordinates into their corresponding NAD 83 coordinates in the following manner (Craymer et al. 2000). Representatives from these two organizations estimated the "best-fitting" values for the seven constants- $T_{x}\left(t_{0}\right), T_{y}\left(t_{0}\right), \ldots, s\left(t_{0}\right)$-for transforming the ITRF96 coordinates of 12 selected very-longbaseline-interferometry (VLBI) stations at $t_{0}=1997.00$ to their corresponding NAD 83 coordinates at this same time (Fig. 1). They subsequently set $s\left(t_{0}\right)$ to zero to ensure that NAD 83 would have the same scale as ITRF96. In addition, they adopted values for the three rotation rates- $\dot{\varepsilon}_{x}, \dot{\varepsilon}_{y}$, and $\dot{\varepsilon}_{z}-$ so as to equal those describing the average motion of the North American tectonic plate according to the "no-net-rotation" NUVEL-1A geophysical model of DeMets et al. (1994). The use of this model is consistent with the IERS convention [McCarthy (1996), Table 3.2]. Finally, they set the values of the remaining four constants $-\dot{T}_{x}, \dot{T}_{y}, \dot{T}_{z}$, and $\dot{s}$ - to zero. Table 1, fifth column, presents the adopted values for the constants for this transformation from ITRF96 to NAD 83. Let us use the notation (ITRF96 $\rightarrow$ NAD83) in referring to this transformation.

Based on the 12 VLBI stations, this transformation was subsequently used to compute NAD 83 coordinates for all CORS sites to establish a new "realization" of NAD 83, technically denoted NAD 83 (CORS96). However, when the context is clear, and to simplify the text, this nomenclature will be truncated in this article to NAD 83. Snay and Soler (2000a) describe the evolution of NAD 83 from its original realization, denoted NAD 83 (1986), to NAD 83 (HARN) and then to NAD 83 (CORS96). The definition of all these realizations remains consistent with the definition of NAD 83 (1986), yet positional coordinates for the same station may differ between any pair of realizations due to the periodic implementation of advancements in technology and computational procedures. That is, NAD 83 (CORS96) represents 


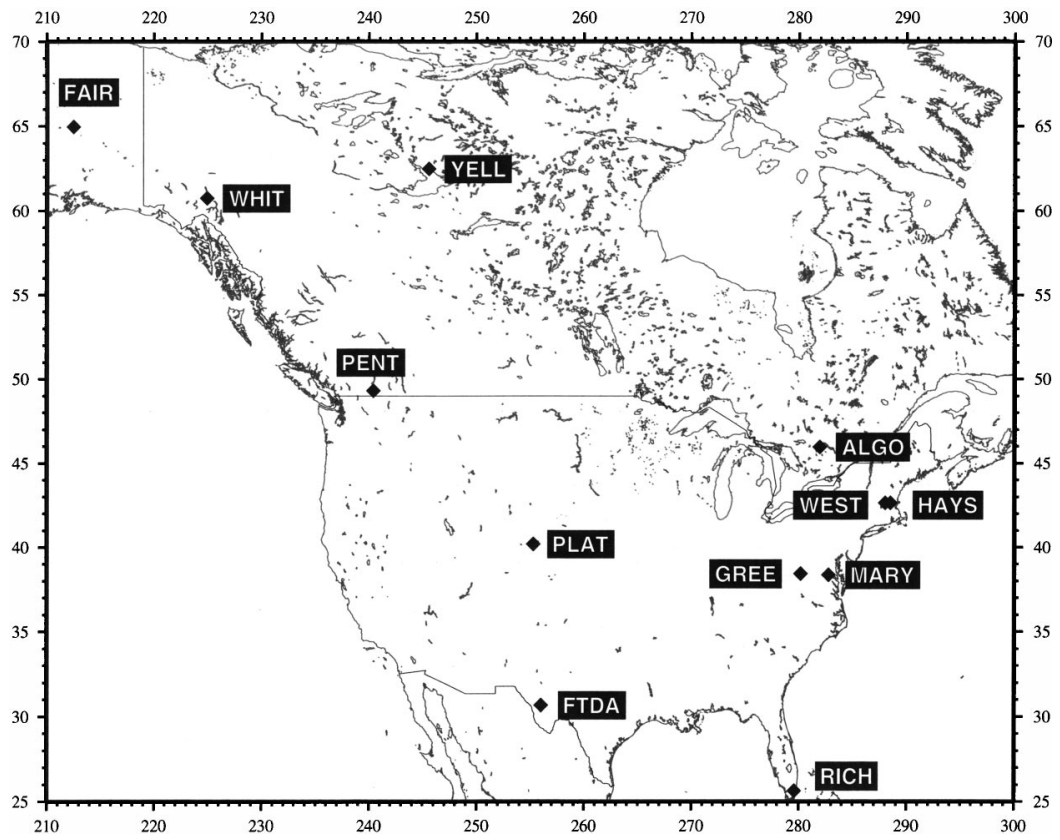

Fig. 1. Twelve very-long-baseline-interferometry stations used to determine transformation ITRF96 $\rightarrow$ NAD83

an improvement of the original realization, NAD 83 (1986). Schwarz (1989) provides detailed information about the definition and establishment of NAD 83 (1986).

Since the adoption of the (ITRF96 $\rightarrow$ NAD 83) transformation, the International Earth Rotation Service (IERS) has introduced two newer realizations, ITRF97 and ITRF00. The transformation of positional coordinates from ITRF00 to NAD 83, denoted (ITRF00 $\rightarrow$ NAD 83), is defined in terms of the composition of three distinct transformations, applied sequentially. First, one transforms positional coordinates from ITRF00 to ITRF97, then from ITRF97 to ITRF96, and finally from ITRF96 to NAD 83. This composition may be symbolically expressed via Eq. (3):

$$
\begin{aligned}
(\mathrm{ITRF} 00 \rightarrow \mathrm{NAD} 83)= & (\text { ITRF00 } \rightarrow \text { ITRF97 }) \\
& +(\text { ITRF97 } \rightarrow \text { ITRF96 }) \\
& +(\text { ITRF96 } \rightarrow \text { NAD 83 })
\end{aligned}
$$

where (ITRF00 $\rightarrow$ ITRF97) and (ITRF97 $\rightarrow$ ITRF96) denote the adopted transformations from ITRF00 to ITRF97 and from ITRF97 to ITRF96, respectively. These adopted transformations are discussed in the following paragraphs.

When ITRF97 was introduced, some controversy as to its relationship with ITRF96 arose. According to the IERS, the organization responsible for defining the various ITRFxx realizations, the "best-fitting" Helmert transformation from ITRF97 to ITRF96 is the identity function (that is, all 14 Helmert parameters are zero in value). Indeed, the IERS defined ITRF97 positional coordinates and velocities for several hundred stations, each of which had been positioned by one or more space-based geodetic techniques, so that they would agree on average with their corresponding ITRF96 positional coordinates and velocities (Boucher et al. 1999).

However, when the IGS independently estimated values for the 14 parameters to transform ITRF97 positional coordinates and

Table 1. Transformation Parameters between Different Frames for $t_{0}=1997.00$

\begin{tabular}{lcccc}
\hline Parameter & Units & ITRF00 $\rightarrow$ ITRF97 & ITRF97 $\rightarrow$ ITRF96 & -0.00207 \\
\hline$T_{x}\left(t_{0}\right)$ & meters & +0.0067 & +0.00069 & ITRF96 $\rightarrow$ NAD 83 \\
$\dot{T}_{x}$ & meters/year & +0.0000 & -0.00021 & +0.9910 \\
$T_{y}\left(t_{0}\right)$ & meters & +0.0061 & -0.00010 & -1.9072 \\
$\dot{T}_{y}$ & meters/year & -0.0006 & +0.00995 & $+0.0^{\mathrm{a}}$ \\
$T_{z}\left(t_{0}\right)$ & meters & -0.0185 & +0.00186 & -0.5129 \\
$\dot{T}_{z}$ & meters/year & -0.0014 & +0.12467 & $+0.0^{\mathrm{a}}$ \\
$\varepsilon_{x}\left(t_{0}\right)$ & mas & $+0.0^{\mathrm{a}}$ & +0.01347 & +25.79 \\
$\dot{\varepsilon}_{x}$ & mas/year & $+0.0^{\mathrm{a}}$ & -0.22355 & +0.0532 \\
$\varepsilon_{y}\left(t_{0}\right)$ & mas & $+0.0^{\mathrm{a}}$ & -0.01514 & +0.06065 \\
$\dot{\varepsilon}_{y}$ & mas/year & $+0.0^{\mathrm{a}}$ & -0.0606 & +0.7423 \\
$\varepsilon_{z}\left(t_{0}\right)$ & mas & $+0.0^{\mathrm{a}}$ & +0.00027 & -0.93496 \\
$\dot{\varepsilon}_{z}$ & mas/year & -0.02 & -0.19201 \\
$s\left(t_{0}\right)$ & ppb & +1.55 & -0.0316 \\
$\dot{s}$ & ppb/year & +0.01 & $+0.0^{\mathrm{a}}$ \\
\hline
\end{tabular}

Note: mas $\equiv$ milliarc second. Counterclockwise rotations of axes are assumed positive; $1 \mathrm{ppb}=10^{-3} \mathrm{ppm}$.

${ }^{a}$ Values set to zero by definition. 
Table 2. Parameters Adopted for Transformation ITRF00 $\rightarrow$ NAD 83 (CORS96)

\begin{tabular}{lccc}
\hline Parameter epoch: & & & \\
$t_{0}=1997.00$ & Definition & Units & Values at $t_{0}$ \\
\hline$T_{x}\left(t_{0}\right)$ & $x$-shift & meters & +0.9956 \\
$T_{y}\left(t_{0}\right)$ & $y$-shift & meters & -1.9013 \\
$T_{z}\left(t_{0}\right)$ & $z$-shift & meters & -0.5215 \\
$\varepsilon_{x}\left(t_{0}\right)$ & $x$-rotation & mas & +25.915 \\
$\varepsilon_{y}\left(t_{0}\right)$ & $y$-rotation & mas & +9.426 \\
$\varepsilon_{z}\left(t_{0}\right)$ & $z$-rotation & mas & +11.599 \\
$s\left(t_{0}\right)$ & scale & ppb & +0.62 \\
$\dot{T}_{x}$ & $x$-shift rate & meters/year & +0.0007 \\
$\dot{T}_{y}$ & $y$-shift rate & meters/year & -0.0007 \\
$\dot{T}_{z}$ & $z$-shift rate & meters/year & +0.0005 \\
$\dot{\varepsilon}_{x}$ & $x$-rotation rate & mas/year & +0.067 \\
$\dot{\varepsilon}_{y}$ & $y$-rotation rate & mas/year & -0.757 \\
$\dot{\varepsilon}_{z}$ & $z$-rotation rate & mas/year & -0.051 \\
$\dot{s}$ & scale rate & ppb/year & -0.18 \\
\hline
\end{tabular}

Note: mas $\equiv$ milliarc second. Counterclockwise rotation of axes are assumed positive; $1 \mathrm{ppb}=10^{-3} \mathrm{ppm}$.

velocities to their ITRF96 counterparts using about 50 wellestablished GPS base stations distributed around the world, this organization found some values that differed significantly from zero (Springer et al. 2000). Hence, while ITRF97 and ITRF96 may be essentially equivalent for a combination of high-precision geodetic data types-VLBI, satellite laser ranging (SLR), and Doppler orbitography and radio-positioning integrated by satellite (DORIS) - the two reference frames are not equivalent when using only GPS observations.

In contrast to ITRF frames, IGS-derived geodetic frames are exclusively based on GPS observations. NRCan and the NGS decided to adopt the IGS-derived transformation from ITRF97 to ITRF96 because most of the precise geodetic work being performed in North America is based on GPS technology. Table 1, fourth column, presents the 14 parameters adopted by the NGS, which equal the original parameters derived by IGS scientists. These values differ, however, from those appearing in Springer et al. (2000) because they correspond to $t_{0}=1997.00$, whereas those in Springer et al. (2000) correspond to $t_{0}=1999.58$ (August 1, 1999). Also, the value of $\dot{\varepsilon}_{z}$ in Springer et al. (2000) differs slightly from the original value derived by IGS investigators.

Similarly, when ITRF00 was introduced (Altamimi et al. 2002), the IERS and IGS assigned different values to the 14 parameters in the transformation from ITRF00 to ITRF97. Again, the IERS values were based on several hundred stations and involved various space-based geodetic observational techniques, whereas the IGS values were based on about 50 GPS base stations. In this case, however, the IERS and IGS estimates for the 14 parameters did not differ significantly in a statistical sense. Hence, NRCan and the NGS adopted the IERS values because the IERS shoulders primary responsibility for defining the ITRFxx realizations. Table 1, third column, presents the IERS values for the 14 parameters for the transformation from ITRF00 to ITRF97. See also ftp://lareg.ensg.ign.fr/pub/itrf/ITRF.TP.

Because the values for the 14 parameters of the three adopted transformations are rather small in magnitude, the values for the 14 parameters for transforming ITRF00 positional coordinates to NAD 83 positional coordinates may be computed with sufficient accuracy by adding across the rows in Table 1 . After some round- ing, the values adopted for transforming ITRF00 $\rightarrow$ NAD 83 (CORS96) at epoch $t_{0}=1997.00$ are given in Table 2 .

A cursory check of the shifts in Table 2 shows that the origin of the NAD 83 frame is offset from the geocenter (as defined by ITRF00) by approximately $2 \mathrm{~m}$ in the $y$ component. This fact makes it imperative that all GPS processing should be computed initially on a more geocentric frame such as ITRF00, and after the very last processing phase, transform the positions from ITRF00 to the NAD 83 (CORS96) frame after substituting in Eq. (1) the adopted parameters of Table 2.

Eq. (1), in conjunction with the 14 parameters just described, has been implemented into the NGS software utility, HTDP (horizontal time-dependent position). This software is freely available at the NGS Web site (http://www.ngs.noaa.gov) by clicking on $\langle\langle$ Geodetic Tool Kit $\rangle$, and then on $\langle\langle$ HTDP $\rangle$. The software enables people to transform positional coordinates from one reference frame to another and/or from one epoch date to another. Individual positions may be entered interactively or a collection of positions entered as a formatted file. When people expect to transform only a few positions, then they may run HTDP interactively from the Web page following the stated instructions. Further technical information about HTDP is discussed in (Snay 1999).

The NGS recognized the implications and promise of the GPS in its early stages of development and embarked on the promotion and adaptation of GPS methods to improve the NSRS. However, this endeavor is an iterative process marked by the everyday demand of improving positional accuracies, constantly guided by the latest advancements in technology and the most recent definitions of coordinate systems. A history of various sets of the NGS-adopted transformation parameters between earlier realizations of ITRF and NAD 83 is available on the NGS Web page (http://www.ngs.noaa.gov/CORS/metadata1).

A further consideration should be kept in mind: The transformation parameters necessary to obtain NAD 83 coordinates from ITRF00 are the result of comparing coordinates at 12 VLBI stations. However, when GPS-derived geodetic networks such as the Federal Base Network (FBN) or the older High Accuracy Reference Network (HARN) are adjusted, some observational errors are propagated, and small distortions appear when coordinates obtained using ITRF00 and the strict application of Eq. (1) are contrasted with the results of the FBN/HARN network adjustment. These differences (up to $6 \mathrm{~cm}$ in the horizontal components) are also influenced by the fact that FBN/HARN results are derived from an ensemble of discrete statewide networks that were planned, observed, and adjusted somewhat independently. For this reason, a homogeneous continentwide adjustmentincluding GPS observations for all A- and B-order reference stations stored in the NGS database-is planned to be performed around 2005.

\section{NAD 83 Velocities}

The advancement of GPS technology and methods has reached the point that, without much effort, relative positioning of stations with respect to the National CORS sites can be accomplished with an accuracy of a few centimeters. Accordingly, in March 2002, the NGS upgraded NAD 83 (CORS96) positional coordinates and velocities for all CORS sites, except those located on islands in the western Pacific Ocean, so that they equal the transformed values of their recently computed ITRF00 positional coordinates and velocities. The special case of the Pacific islands is treated at length by Snay (2003). This upgrade removed inconsis- 
tencies among the previously published NAD 83 (CORS96) positional coordinates and velocities for the CORS. In some cases, these positional inconsistencies exceeded $2 \mathrm{~cm}$, horizontally, and/or $4 \mathrm{~cm}$, vertically. Such inconsistencies can be easily detected with current high accuracy GPS surveys. These inconsistencies would have corrupted, for example, results obtained with the On-line Positioning User Service (OPUS), a Web-based utility that uses CORS positions and velocities to compute accurate positions for other points (Mader et al. 2003).

Moreover, for each CORS site, the NGS is now providing NAD 83 (CORS96) positional coordinates that are referenced to an epoch date of 2002.00. That is, the published coordinates for a site's position correspond to this site's location on January 1, 2002. Special attention should be given to apply NAD 83 (CORS96) site velocities when computing NAD 83 site positions at any other date. Previously, NAD 83 (CORS96) positions for the CORS sites were published for the epoch date of 1997.00 (January 1, 1997). The use of a more current epoch date will reduce those systematic errors introduced when points are positioned relative to CORS sites at different epochs without appropriately applying their site velocities.

This more current epoch date will especially benefit those people involved in positioning activities in areas of active crustal motion, such as the western Continental United States and Alaska. On the other hand, published ITRF00 positional coordinates will continue to be referenced to an epoch date of 1997.00 to remain consistent with international convention. Generally speaking, geographic points located in the eastern and central United States have NAD 83 velocities that are close to zero, and therefore their NAD 83 positional coordinates should not change much between different epochs. This is not the case for geographic points in the United States that are located within a few hundred kilometers of the Pacific Coast; here NAD 83 horizontal velocities with magnitudes in excess of $5 \mathrm{~mm} /$ year are prevalent.

Velocities referred to the NAD 83 (CORS96) reference frame can be obtained from their corresponding ITRF00 velocities by taking the derivatives of Eq. (1) with respect to time. After neglecting second-order terms, the following equations are obtained:

$$
\begin{aligned}
& v_{x_{\mathrm{NAD} 83}}=v_{x_{\mathrm{ITRF}}}+\dot{T}_{x}+\dot{s} \cdot x(t)_{\mathrm{ITRF}}+\dot{\omega}_{z} \cdot y(t)_{\mathrm{ITRF}}-\dot{\omega}_{y} \cdot z(t)_{\mathrm{ITRF}} \\
& v_{y_{\mathrm{NAD} 83}}=v_{y_{\mathrm{ITRF}}}+\dot{T}_{y}+\dot{s} \cdot y(t)_{\mathrm{ITRF}}-\dot{\omega}_{z} \cdot x(t)_{\mathrm{ITRF}}+\dot{\omega}_{x} \cdot z(t)_{\mathrm{ITRF}} \\
& v_{z_{\mathrm{NAD} 83}}=v_{z_{\mathrm{ITRF}}}+\dot{T}_{z}+\dot{s} \cdot z(t)_{\mathrm{ITRF}}+\dot{\omega}_{y} \cdot x(t)_{\mathrm{ITRF}}-\dot{\omega}_{x} \cdot y(t)_{\mathrm{ITRF}}
\end{aligned}
$$

where

$$
\begin{aligned}
& \dot{\omega}_{x}=\dot{\varepsilon}_{x} \cdot m_{r} \\
& \dot{\omega}_{y}=\dot{\varepsilon}_{y} \cdot m_{r} \\
& \dot{\omega}_{z}=\dot{\varepsilon}_{z} \cdot m_{r}
\end{aligned}
$$

Note that in Eq. (4), and according to Table 2, the magnitudes of $\dot{T}_{x}, \dot{T}_{y}$, and $\dot{T}_{z}$ do not exceed $0.7 \mathrm{~mm} \cdot$ year $^{-1}$; and similarly, the products of $\dot{s}$ and any component of the positional coordinates do not exceed $1.1 \mathrm{~mm} \cdot$ year $^{-1}$ in magnitude on the surface of the Earth. Eq. (4), in conjunction with the set of adopted transformation parameters, is implemented in the NGS software, HTDP, to compute velocities of the CORS sites referred to NAD 83 (CORS96). Therefore, CORS sites have coordinates and velocities referred to the NAD 83 (CORS96) reference frame, and the transformation of NAD 83 coordinates from epoch to epoch is possible.
The situation is different when one deals with coordinates of the FBN/HARN marks that are also retrievable from the NGS database. First of all, it should be pointed out that these coordinates implicitly refer to the frame and corresponding epoch that were in use when the statewide or regional network adjustment was completed. At that particular moment, one or several CORS sites included in the network were constrained using the thenavailable frame and epoch. However, the NGS does not provide a velocity for an FBN/HARN mark on its "data sheet" (see examples of data sheets at 〈http://www.ngs.noaa.gov/ datasheet.html ) ) because, generally, insufficient observations exist to estimate this velocity with good accuracy. Data sheets are the NGS's principal informational product. Each data sheet provides positional and descriptive information for a specific geodetic station in the NSRS. NGS data sheets can be retrieved directly from the NGS database.

HTDP-predicted NAD 83 velocities are zero if the area where the point is located is free from crustal motion activity, for example, most of the eastern United States. However, some geodetic marks located in the western United States may need to be updated to epoch 2002.00 before they are used for GPS densification work. In this instance, it is possible to update FBN/HARN NAD 83 point coordinates to epoch 2002.00 using its NAD 83 velocity. NAD 83 velocity components $v_{x_{\mathrm{NAD}} 83}, v_{y_{\mathrm{NAD}} 83}$, and $v_{z_{\mathrm{NAD}} 83}$ at any FBN/HARN mark can be predicted using the NGS software HTDP available from the Web. From the NGS main page (http://www.ngs.noaa.gov/), click sequentially along the following path: 《UGeodetic Tool Kit $\rangle\rangle \rightarrow\langle\langle$ HTDP $\rangle\rangle$ $\rightarrow\langle\langle$ Interactive Computations $\rangle\rangle \rightarrow\langle\langle$ Interactive Predict Velocities $\rangle\rangle$ $\rightarrow\langle\langle$ Individual Points Entered Interactively $\rangle \rightarrow\langle\langle$ NAD 83 $\rangle$. . These velocities could then be applied to update FBN/HARN NAD 83 (CORS96) positions to epoch 2002.00 as follows:

$$
\begin{aligned}
& x_{\mathrm{NAD} 83}(2002.00) \approx x_{\mathrm{NAD} 83}\left(t_{F}\right)+v_{x_{\mathrm{NAD} 83}} \cdot\left(2002.00-t_{F}\right) \\
& y_{\mathrm{NAD} 83}(2002.00) \approx y_{\mathrm{NAD} 83}\left(t_{F}\right)+v_{y_{\mathrm{NAD} 83}} \cdot\left(2002.00-t_{F}\right) \\
& z_{\mathrm{NAD} 83}(2002.00) \approx z_{\mathrm{NAD} 83}\left(t_{F}\right)+v_{z_{\mathrm{NAD} 83}} \cdot\left(2002.00-t_{F}\right)
\end{aligned}
$$

where $t_{F}$ is the epoch of the FBN/HARN adjustment as given on the NGS data sheet for this station.

Eq. (6) has been encoded into HTDP so that users of that software can compute positional coordinates at epoch 2002.00 simply by entering the positional coordinates at epoch $t_{F}$. Actually, the code in HTDP is more general than Eq. (6) in four ways. First, users can apply HTDP to update the positional coordinates to any arbitrary epoch $t$, not just to epoch 2002.00. Second, HTDP enables its users to enter the positional coordinates at time $t_{F}$ in terms of $x, y$, and $z$ or in terms of their equivalent latitude, longitude, and ellipsoidal height. HTDP also provides the resulting positional coordinates at epoch 2002.00 (or epoch $t$ ) both in terms of $x, y$, and $z$ and in terms of latitude, longitude, and ellipsoidal height. Third, HTDP provides its users the option of entering their own values for $v_{x_{\mathrm{NAD}} 83}, v_{y_{\mathrm{NAD} 83}}$, and $v_{z_{\mathrm{NAD} 83}}$ instead of using the values predicted by the crustal motion model that has been encoded into HTDP. Fourth, HTDP includes mathematical models for predicting those episodic motions associated with certain major earthquakes. Eq. (6) is based on the assumption that no such episodic motions have occurred between epoch $t_{F}$ and epoch 2002.00. For all these reasons, it is recommended that HTDP be used to transform positional coordinates from one epoch date to another. 


\section{OPUS Transformations}

In March 2001, NGS introduced the OPUS Web-based utility. OPUS enables its users to submit static GPS data to the NGS via the World Wide Web, whereby OPUS will compute the positional coordinates for the location associated with these data. OPUS uses NGS computers and PAGES (program for the adjustment of GPS ephemeris http://www.grdl.noaa.gov/GRD/GPS/DOC/ toc.html) software. OPUS processes the submitted data together with GPS data from three National CORS sites, and the user receives the derived positional coordinates via e-mail in a timely fashion, usually a few minutes. The following steps outline how OPUS currently obtains ITRF00 coordinates and then applies the (ITRF00 $\rightarrow$ NAD 83) transformation to obtain corresponding NAD 83 (CORS96) coordinates:

- ITRF epoch transformation: After identifying three "suitable" CORS, OPUS retrieves their adopted ITRF00 positional coordinates and velocities from the NGS Integrated Data Base (NGSIDB). Because the NGSIDB stores these ITRF00 positional coordinates for an epoch date of 1997.00, OPUS uses the stored velocities to calculate the corresponding ITRF00 positional coordinates for the midpoint of the time interval during which the submitted data were observed (epoch $t$ ).

- PAGES software invoked: Using the submitted GPS data in conjunction with the publicly available GPS data for the three selected CORS, OPUS invokes PAGES software to determine three independent sets of ITRF00 positional coordinates (epoch $t$ ) and their corresponding intersite vector components $(\Delta x, \Delta y, \Delta z)$, also at epoch $t$. All these values are computed using the GPS data from each CORS site while holding fixed its coordinates determined in the previous step.

- ITRF positions averaged: The above three positions are averaged to obtain the final ITRF00 coordinates of the unknown point at epoch $t$.

- Frame transformation: The ITRF00 intersite vector components $(\Delta x, \Delta y, \Delta z)$ computed by PAGES are then transformed to the NAD 83 (CORS96) frame using equations similar to Eq. (1). In fact, these equations have the same form and values as Eq. (1), except $T_{x}(t)=T_{y}(t)=T_{z}(t)=0$ (Soler et al. 2002). Notice that the resulting NAD 83 intersite vector components still refer to epoch $t$, the midpoint of the time interval when the data were observed.

- NAD 83 epoch transformation: OPUS retrieves NAD 83 (CORS96) positional coordinates and velocities for the three CORS from the NGSIDB. Because the NGSIDB stores these positional coordinates for an epoch date of 2002.00, OPUS uses an equation in the form of Eq. (6), to determine the corresponding NAD 83 positional coordinates at epoch $t$, the midpoint of the time interval when the submitted GPS data were observed.

- Three NAD 83 positions computed: Each NAD 83 intersite vector is added to the NAD 83 coordinates of the corresponding CORS, as determined in the previous step, to obtain three sets of NAD 83 positional coordinates for the unknown point at epoch $t$, the midpoint of the data time interval.

- NAD 83 positions averaged: The average of the above three values is adopted as the NAD 83 positional coordinates of the unknown point at epoch $t$.

- HTDP model invoked: Finally, OPUS invokes the HTDP software to transform the above NAD 83 positional coordinates to epoch 2002.00. This step requires HTDP to predict the NAD 83 velocity for the unknown point and to apply this velocity in a manner similar to Eq. (6).
Note that OPUS-derived NAD 83 (CORS96) positional coordinates are not obtained by a direct frame transformation of their corresponding ITRF00 coordinates, but by the sequence of steps described above.

\section{Conclusions}

Positional coordinates and velocities of an arbitrary point may be transformed from ITRF00 to NAD 83 using Eqs. (1) and (4), respectively. The NGS applied this technique in March 2002 to update NAD 83 position coordinates for all CORS sites so that these coordinates are consistent with adopted ITRF00 coordinates. Furthermore, the NGS updated the NAD 83 coordinates for all CORS to an epoch date of 2002.00. This latter update makes it easier for GPS users working in regions prone to crustal motions to obtain accurate results when site velocities are not appropriately applied. CORS positions effectively act as both ITRF and NAD 83 fiducial (reference) points from which accurate GPS networks could be propagated and densified. A new NSRS framework based on the most accurate CORS coordinates and velocities is expected to be completed by 2005 , perpetuating the primary objective of the NGS: to provide and maintain a spatial reference frame for all surveying and mapping applications in the United States.

\section{Acknowledgments}

The writers wish to express their sincere thanks to C. Goad, J. Marshall, D. Milbert, and B. H. W. van Gelder for their thoughtful review and valuable suggestions.

\section{References}

Altamimi, Z., Sillard, P., and Boucher, C. (2002). "ITRF2000: A new release of the International Terrestrial Reference Frame for Earth science applications." J. Geophys. Res., 107(B10), ETG2/1-19.

Boucher, C., and Altamimi, Z. (1996). "International Terrestrial Reference Frame." GPS World, 7(9), 71-74.

Boucher, C., Altamimi, Z., and Sillard, P. (1999). "The International Terrestrial Reference Frame (ITRF97)." Intern. Earth Rotation Serv. Tech Note 27, Observatoire de Paris, Paris.

Craymer, M., Ferland, R., and Snay, R. (2000). "Realization and unification of NAD 83 in Canada and the U.S. via the ITRF." In Towards an Integrated Global Geodetic Observing System (IGGOS), R. Rummel, H. Drewes, W. Bosch, and H. Hornik, eds., IAG Section II Symp., International Association of Geodesy Symposia, Vol. 120, Springer, Berlin, 118-121.

DeMets, C., Gordon, R. G., Argus, D. F., and Stein, S. (1994). "Effect of recent revisions to the geomagnetic reversal time scale on estimates of current plate motions." Geophys. Res. Lett., 21(20), 2191-2194.

Eckl, M. C., Snay, R. A., Soler, T., Cline, M. W., and Mader, G. L. (2001). "Accuracy of GPS-derived relative positions as a function of interstation distance and observing-session duration." J. Geodesy, Berlin, 75(12), 633-640.

Mader, G. L., Weston, N. D., Morrison, M. L., and Milbert, D. G. (2003). "The On-line Positioning User Service (OPUS)." Prof. Surv., 23(5), $26,28,30$.

McCarthy, D. D., ed. (1996). "IERS Conventions (1996)." Technical Note 21, International Earth Rotation Service, Paris Observatory, Paris.

Merrigan, M. J., Swift, E. R., Wong, R. F., and Saffel, J. T. (2002). “A refinement to the World Geodetic System 1984 Reference Frame." 
Proc., ION GPS 2002 (CD-ROM), Institute of Navigation, Alexandria, Va., 1519-1529.

Schwarz, C. R., ed. (1989). "North American Datum of 1983." NOAA Professional Paper NOS 2, U.S. Dept. of Commerce, National Oceanic and Atmospheric Administration, Silver Spring, Md.

Snay, R. A. (1999). "Using the HTDP software to transform spatial coordinates across time and between reference frames." Surv. Land Inf. Sys., 59(1), 15-25.

Snay, R. A. (2003). "Introducing two spatial reference frames for regions of the Pacific Ocean." Surv. Land Inf. Sci., 63(1), 5-12.

Snay, R. A., Adams, G., Chin, M., Frakes, S., Soler, T., and Weston, N. D. (2002). "The sinergistic CORS program continues to evolve." Proc., ION GPS 2002 (CD-ROM), Institute of Navigation, Alexandria, Va., 2630-2639.

Snay, R. A., and Soler, T. (2000a). "Modern terrestrial reference systems. Part 2: The evolution of the NAD 83." Prof. Surv., 20(2), 1-2.
Snay, R. A., and Soler, T. (2000b). "Modern terrestrial reference systems. Part 3: WGS84 and ITRS." Prof. Surv., 20(3), 1-3.

Soler, T., and Hothem, L. D. (1988). "Coordinate systems used in geodesy: Basic definitions and concepts.” J. Surv. Eng., 114(2), 84-97.

Soler, T., and Hothem, L. D. (1989). "Important parameters used in geodetic transformations." J. Surv. Eng., 115(4), 414-417.

Soler, T., and Marshall, J. (2003). "A note on frame transformations with applications to geodetic datums." GPS Solutions, 7(1), 23-32.

Soler, T., Weston, N. D., and Han, H. (2002). "Computing NAD 83 coordinates using ITRF-derived vector components." Proc., XII FIG Int. Congress, ACSM/ASPRS, Washington, D.C.

Springer, T. A., and Hugentobler, U. (2001). "IGS ultra rapid products for (near-) real-time applications." Phys. Chem. Earth, 26(6-8), 623628.

Springer, T. A., Kouba, J., and Mireault, Y. (2000). “1999 analysis coordinator report." 1999 Tech. Rep., International GPS Service for Geodynamics, Jet Propulsion Laboratory, Pasadena, Calif., 15-55. 